Horizons philosophiques

\title{
La facticité du domaine numérique vue à travers les lunettes du Tractatus de Wittgenstein
}

\section{Alain Sondheim}

Volume 6, numéro 2, printemps 1996

La philosophie sur Internet

URI : https://id.erudit.org/iderudit/801009ar

DOI : https://doi.org/10.7202/801009ar

Aller au sommaire du numéro

Éditeur(s)

Collège Édouard-Montpetit

ISSN

1181-9227 (imprimé)

1920-2954 (numérique)

Découvrir la revue

Citer cet article

Sondheim, A. (1996). La facticité du domaine numérique vue à travers les lunettes du Tractatus de Wittgenstein. Horizons philosophiques, 6(2), 49-54. https://doi.org/10.7202/801009ar d'utilisation que vous pouvez consulter en ligne.

https://apropos.erudit.org/fr/usagers/politique-dutilisation/ 


\section{LA FACTICITÉ DU DOMAINE \\ NUMÉRIQUE VUE À TRAVERS \\ LES LUNETTES DU TRACTATUS \\ DE WITTGENSTEIN*}

Il est toujours bon de revenir aux premières sources de la pensée, en réexaminant par exemple les propositions du Tractatus logico-philosophicus, à la lumière de considérations provenant du domaine numérique. On sait que le travail de Wittgenstein repose sur la «barre de Sheffer» («non p ou non q») et son double, la négation simultanée («ni a nib»), permettant toutes deux de construire toute fonction de vérité au moyen d'une seule opération, appliquée successivement à toutes les propositions élémentaires. Or le doublet négation alternative/ négation simultanée a son équivalent dans le domaine numérique, qui est un calcul booléen utilisant les valeurs 0 et 1 . Font partie du domaine numérique toutes les constructions numériques, incluant ASCII, Mosaic, Quick-Time, etc.

La barre de Sheffer et son double servent à construire l'antinégation, qui est AILLEURS. L'instabilité de la barre de Sheffer est renforcée par le «ni a ni b», qui renvoie à un domaine fissuré et non circonscrit. Si la barre de Sheffer est une réorganisation du calcul propositionnel à partir d'une seule base, la négation simultanée est une ouverture au-delà de ce calcul (ailleurs).

Àl'intérieur d'un environnementinformatique ou d'un réseau télématique, les deux opérations font constamment appel au domaine numérique au niveau de l'adresse, du protocole ou de la reconnaissance. On a ici l'image d'un opéra, joué par la barre de Sheffer surles symboles ASCII (quelque chose de comparable à une expérimentation de pensée où on imaginerait que des singes puissent écrire la pièce «Hamlet»); dès lors, tous les énoncés ASCII, qui sont toujours déjà codés, sont reproduits. Le Tractatus serait valable dans ce domaine limité, la table de

* Tiré de l'Internet Text, vol. 4, d'Alain Sondheim : http://jefferson.village.virginia.edu/ spoons/internet_txt.html 
vérité se trouvant être l'existence d'une chaîne ASCIl à l'intérieur d'une structure préalable. Ainsi, dans le corps de cet essai, l'énoncé ASCII "énoncé ASCII» égale 1, puisqu'il est actuellement présent.

Il est clair que des ensembles d'énoncés potentiels sont des sous-ensembles d'autres ensembles d'énoncés, ce qui signifie que le domaine du digital se replie sur lui-même. Notons que si on retient l'argument de la diagonale de Cantor, il ne serait jamais possible, même théoriquement, au niveau aleph zéro, de reproduire l'ensemble de tous les énoncés. Ce que nous considérons ici est plutôt l'ensemble de tous les énoncés qui sont toujours déjà produits à l'intérieur d'un domaine, par exemple la totalité des énoncés exprimés sur le W3 de 1990 à 1995. Le domaine numérique est déterminé du point de vue de la quantité d'information binaire; toutefois, on peut envisager une construction plus profonde, au-delà du domaine ASCII, faisant appel à la barre de Sheffer et à la négation simultanée. Cette construction s'appuie uniquement sur la distinction entre 1 et 0 , l'un et l'autre.

On peut relire les sept principales propositions du Tractatus et quelques autres à la lumière de ce qui précède.

1. Le monde est tout ce qui arrive.

\section{Die Welt ist alles, was der Fall ist.}

La virgule qui est présente dans le texte original indique que le monde est une totalité. L'Internet (ou tout autre domaine numérique) est "ce qui arrive» ou "ce qui est le cas", le cas étant défini par le protocole.

\section{Ce qui arrive - un fait- est l'existence des états de choses.}

Traduit dans les termes de la communication digitale : un fait est une concaténation ou une chaîne de symboles binaires. Remarquez que les symboles sont vides; ce qui est évident à partir de 2.01 : «Un état de choses est une combinaison d’objets (de choses et non pas de symboles)." 


\section{Le tableau logique des faits constitue la pensée.}

3.01 La totalité des pensées vraies forme un tableau du monde.

Dans le domaine binaire, «logique» est défini comme «instance de", la barre de Sheffer ou la négation simultanée servant à produire des concaténations de symboles binaires. Ce domaine est techniquement réflexif.

\section{La pensée est la proposition pourvue de sens.}

4.002 L'être humain possède la capacité de construire des langages pouvant exprimer tout ce qui a un sens, sans savoir ce que chaque mot signifie et la manière dont il le fait.

Les énoncés de cette section parlent en faveur d'une division hiérarchique du domaine digital, comme c'est le cas dans la topographie de l'Internet ou à l'intérieur d'un ordinateur, dans l'escalade du langage, de l'assemblage de la machine à la programmation de haut niveau par les compilateurs et interprètes. Wittgenstein dit encore : «Le langage travestit la pensée. À tel point que l'on ne saurait se fier à l'apparence du vêtement pour conclure quelque chose concernant la forme de la pensée, car un vêtement extérieur n'est justement pas fait pour permettre de reconnaître la forme du corps".

Si le logicisme du Tractatus a été considéré comme fautif dans son insistance sur un atomisme métaphysique, l'atomisme en question revient en force dans le domaine numérique. De toute évidence, ce domaine opère TOUJOURS à l'intérieur de limites précises, mais en vertu de la qualité gestuelle de logiques en treillis qui sont non distributives et non booléennes, le domaine lui-même s'étend au-delà de limites précises (AILLEURS). Le retour à Wittgenstein et à son argument n'en constitue pas moins un retour au classicisme du XIXe siècle, un rêve d'absolu. Ironiquement, ce rêve devient aussi celui du futur. 


\section{Une proposition est une fonction de vérité de} propositions élémentaires. (Une proposition élémentaire est une fonction de vérité d'elle-même.)

La première partie de cet énoncé parle en faveur de propositions complexes ou de concaténations de propositions élémentaires (la conception atomiste). La deuxième partie parle en faveur d'une réverbération ou auto-réflexivité de tout énoncé. Or ces considérations sont applicables aux langages informatiques. Dans le langage QBasic par exemple, on utilise les symboles de concaténation «AND» ou «:». Dans ce langage, la proposition « $X=X+1$ » est vraie et opérationnelle à l'intérieur d'un programme, si $X$ est un nombre défini ou un nombre non défini. Toutefois, la proposition « $X+1=X »$ ne fonctionne pas, car ce qu'elle demande n'est pas clair. On pourrait dire qu'une proposition élémentaire est vraie d'elle-même si elle fonctionne, et qu'ellen'est pas vraie (fausse) d'elle-même sielle ne fonctionne pas. Voilà qui permet d'éviter le saut ontologique de la tautologie à la substance. Une autre manière d'éviter ce saut est de considérer l'ensemble des symboles $(0 / 1$, etc.) comme l'ensemble des propositions élémentaires.

6. La forme générale d'une fonction de vérité est :

[l'ensemble de toutes les propositions élémentaires; une sélection qui nous donne l'ensemble des propositions élémentaires de n'importe quelle proposition et qui est fonction de l'ensemble de toutes les propositions élémentaires; la négation des valeurs de cette sélection]. C'est là la forme générale d'une proposition.

La proposition 5.632 est immédiatement applicable : «Le sujet ne fait pas partie du monde; il est plutôt la limite du monde». Il en va de même pour la proposition 5.641 : «lly a donc un sens auquel on peut parler en philosophie du moi d'une manière qui ne soit pas psychologique. Le moi intervient en philosophie du fait que «le monde est mon monde». Le moi philosophique n'est pas l'être humain, ni le corps humain, ni l'âme humaine (objet de la psychologie). Le moi philosophique 
est le sujet métaphysique - la limite du monde plutôt que sa partie."

Le domaine digital est doté d'un INTÉRIEUR - son ordre hiérarchique - et d'un EXTÉRIEUR qui est toujours déjà ailleurs, le sujet métaphysique propulsant la sémantique du domaine. Si le domaine binaire est granulaire, le sujet métaphysique nel'est pas. Mais l'explication du sujet s'approche de la granulation, tout comme la réalité virtuelle produit des images toujours plus fines. II n'y a pas de point ultime ici.

6.5: «Une réponse qui ne peut être exprimée en mots suppose une question qui elle non plus ne peut être exprimée. II n'y a pas d'énigme. Si une question peut être posée, il est également possible d'y répondre". Une question à laquelle on peut répondre est celle qui est formatée. Formater une question, c'est l'écrire en vue de la faire travailler. La représentation du sujet métaphysique est toujours une réécriture à l'intérieur du domaine numérique.

\section{Ce dont on ne peut parler, il faut le taire.}

Cette dernière proposition a donné lieu à de nombreuses interprétations, qui vont de l'expression des limites du domaine de la facticité à une représentation problématique de l'éthique. II n'y a pas de doute que les dernières sections du Tractatus vont dans le sens d'un ethos. Si on adopte la sémantique de Kripke, ce qui est tu n'est jamais une espèce naturelle ni un désignateur rigide. Car on peut nommer ce qui tient dans le monde des tableaux, des faits et des propositions. Le silence reflète plutôt l'extériorité du sujet métaphysique, dont la sémantique réelle ne se réduit pas à la codification (simple, double ou triple). Et pourtant, le domaine numérique estmanipulé et la codification est encore présente, car elle faitnécessairement partie de la définition de la vie, qu'il s'agisse de formes de vie artificielles ou autres.

Qu'on le définisse comme on voudra, le domaine numérique est une construction susceptible de recevoir un traitement analytique fondé sur le Tractatus et d'autres textes similaires. Quant au contenu descriptif du dernier Wittgenstein, 
particulièrement dans les Investigations philosophiques, il applique une épistémologie irrégulière au niveau sémantique du sujet métaphysique. Le rapport entre le premier travail et le dernier est parallèle à la distinction entre intérieur et extérieur. Au début du troisième millénaire, l'importance accrue du digital reflète l'articulation accrue de l'extérieur relativementà l'intérieur. D'où la nécessité d'un retour au premier Wittgenstein, voire même à une mécanique hertzienne. La division du sujet qui en résulte est inévitable : on a l'horizon du contrôle parfait et de la description d'un côté, et de l'autre côté on a la fragmentation postmoderne de la totalité du monde réel à tous les niveaux, de l'État nation aux mathématiques et au domaine numérique. En d'autres termes, la torsion n'a pas lieu uniquement pour une subjectivité postmoderne dispersée, mais en l'absence d'une articulation digitale qui contient un germe du classicisme du $\mathrm{XIXe}$ siècle. Cette articulation serait essentielle à toute forme de communication digitale voire même à toute forme de réalité.

\author{
Alain Sondheim (sondeim @ panix.com) \\ Modérateur des listes Cybermind, \\ Image et Fiction-of-Philosophy
}

Traduction de Josette Lanteigne

\title{
Bibliographie
}

Ludwig Wittgenstein, Tractatus logico-philosophicus, London, Routledge \& Kegan Paul Ltd, 1961.

Ludwig Wittgenstein, Tractatus logico-philosophicus suivi de Investigations philosophiques, Paris, Gallimard, 1961. 